\title{
Sydney Work on 21-cm Observations of the HI Galactic Disk
}

\author{
F. J. KERR* \\ Radiophysics Laboratory, Sydney, Australia
}

$T$

HIS paper reports briefly on the provisional results of the $21-\mathrm{cm}$ galactic work at the Radiophysics Laboratory, Sydney. Observations were made with a 36-ft, transit-type radio-telescope whose beam width at half-power is $1^{\circ} .5$ and a 4-channel receiver whose band width, $40 \mathrm{kcps}$, is equivalent to $8 \mathrm{~km} / \mathrm{sec}$.

The large scale structure of the galaxy has been studied by Kerr, Hindman, and Carpenter. ${ }^{1}$ They derive a general picture of the spiral structure in the southern hemisphere extending the Leiden results (see Fig. 1) and, in addition, a "relief map" of the galaxy showing the location of the densest parts of the hydrogen layer with respect to a central plane (see Fig. 2). Analysis of the spiral structure is for the present limited to regions beyond $3 \mathrm{kpc}$ from the center. The Sydney and Leiden data agree well where they overlap, but location of spiral arms is sensitive to the choice of rotational

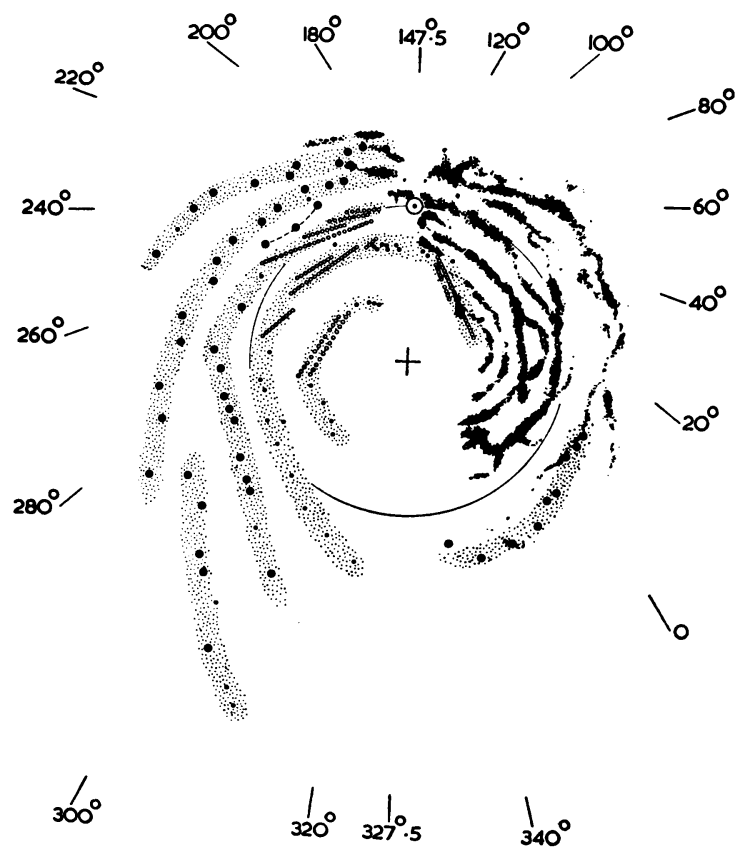

Fig. 1. Composite map of the spiral structure of the galaxy. The Sydney pattern is to the left of the picture, the Leiden one (slightly retouched) to the right, with some overlap. The different character of the two sides results from different methods of analysis and representation. Observed points (Sydney). Less certain points. $\bigcirc \bigcirc \bigcirc$ Extended concentrations. The distance from the sun $(\odot)$ to the galactic center $(+)$ is taken as $8.2 \mathrm{kpc}$.

* Presented by G. de Vaucouleurs.

${ }^{1}$ Kerr, Hindman, and Carpenter, Nature 180, 677-679 (1957). model. Four or five spiral arms, or sections of arms, are indicated in the longitude interval $l=170^{\circ}$ to $l=320^{\circ}$. The arms are clearly trailing in the rotation.

The gas layer, about $250 \mathrm{pc}$ thick at half-density, is remarkably flat in the central regions of the galaxy out to the sun's distance; the sun is in or very close to this "principal plane" including the galactic center at $l=327^{\circ} .5, b=-1^{\circ} .5$ (Harvard Pole). In the outer parts a systematic distortion raises the densest part of the layer up to $+300 \mathrm{pc}$ above the principal plane near $l=40^{\circ}$, and brings it down to $-500 \mathrm{pc}$ or more below the same plane near $l=240^{\circ}-250^{\circ}$, i.e., in the direction of the Large Magellanic Cloud. The effect, however, is about two orders of magnitude too large for a simple gravitational explanation.

The (smoothed) mass distribution of galactic neutral hydrogen has been determined by Kerr and Hindman ${ }^{2}$ and compared with the distribution of total mass given by Schmidt. ${ }^{3}$ The space density of hydrogen is relatively constant beyond 4-5 kpc from the center, but decreases rapidly in the inner regions. As a result both the space

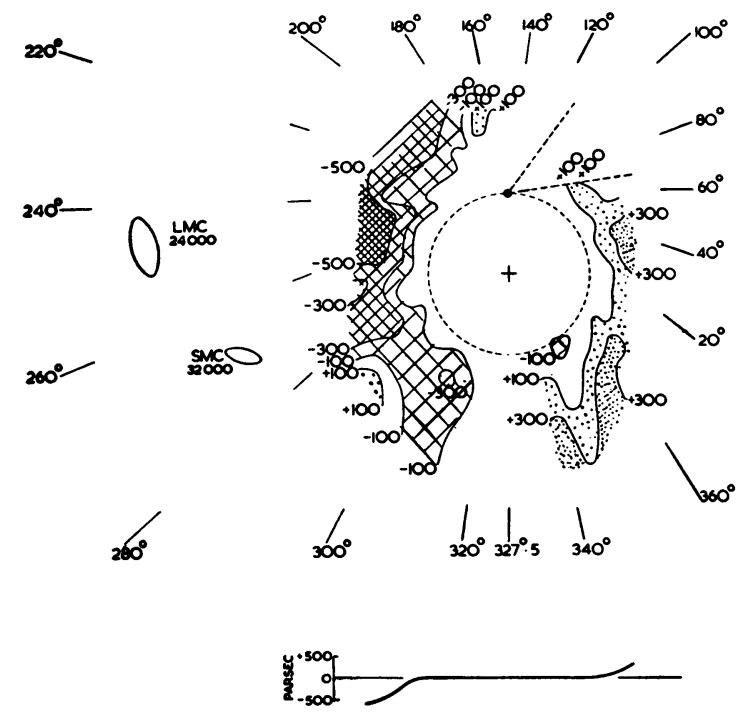

Fig. 2. (a) Relief map of the galaxy, showing departures of the equatorial surface of the hydrogen from an approximation to the principal plane. The contour levels are in parsecs. (b) Cross section through galactic center, in direction of Large Magellanic Cloud.

${ }^{2}$ F. J. Kerr and J. V. Hindman, Publs. Astron. Soc. Pacific 69, 558-560 (1957)

${ }^{3}$ M. Schmidt, Bull. Astron. Soc. Neth. No. 458 (1956). 
density in the equatorial "plane" and the density projected on to the principal plane of the system increase rapidly outwards relative to the total mass density. Near the sun $(R=8 \mathrm{kpc})$ the relative space density of hydrogen is $15 \%$; it is very low near the center and reaches up to $50 \%$ near $R=13 \mathrm{kpc}$. Integration over the whole galaxy gives a ratio of neutral hydrogen to total mass of only $2 \%$; this figure may be but slightly increased if allowance is made for ionized and neutral hydrogen in the galactic corona.

\section{DISCUSSION}

M. A. LEVINE, Advanced Research Laboratory, Geophysics Research Directorate, HQAFCRC, Hanscom Field, Bedford, Massachusetts: Is there any information, or limits, on the amount of ionized and un-ionized material above and below the disk of the galaxy?

G. DE VAUCOULEURS, Lowell Observatory, Flagstaff, Arizona: The thickness of the neutral hydrogen layer at half-density is about $250 \mathrm{pc}$; we do not know about the ionized.

M. P. SAVEDOFF Department of Astronomy, University of Rochester, Rochester, New York: Would you comment on the interpretation of the nonplaneness of the galaxy, which you referred to as a possible tidal effect? Has there been any calculation to show whether an explanation can be given in terms of gravitational interaction with the Small and Large Magellanic Clouds?

G. DE VAUCOULEURS : Kerr has made a rough computation. We know roughly the mass of the large and small clouds. It turns out that the distortion observed, which is up to $+300 \mathrm{pc}$ away from the clouds and down to $-500 \mathrm{pc}$ in the direction of the clouds, is too large by about two orders of magnitude to be explained by gravitational effects. But really I am not surprised. We see such enormous distortions in many galaxies, and bridges between galaxies, and also more fancy things which cannot possibly be explained by gravitation, neither in order of magnitude nor even qualitatively in shape.

V. A. AMBARTSUmian, Burakan Astrophysical Observatory, Academy of Sciences of the Armenian S.S.R., Erevan, U.S.S.R.: You have given the $Z$ coordinates of the surface of the maximum density of the neutral hydrogen in the galaxy, which gives the deviation from the galactic plane. Have you any data about the mean value of $Z$ ? I understand that you have not calculated this and more data are necessary to calculate the mean values of $Z$ for the whole layer. But do you assert that the mean values also will approximately follow the pattern which is obtained for the maximum density?

G. DE VAUCOULEURS : Kerr, as I understand, has determined the plane of maximum density. He had tracings across the galactic plane, and he determined the $Z$ coordinate of maximum space density at various distances, not including the less dense regions as weighted by the density. However, since the layer is only $250 \mathrm{pc}$ thick, the weighted mean cannot be far from the maximum.

J. M. BURGERS, University of Maryland, College Park, Maryland: In your sketch, the central part of the galaxy was rather plane. Are you quite sure about that? Is it only tilted beyond a certain distance from the center?

G. DE VAUCOULEURS : I hope I am not misinterpreting the Sydney data, but I understood that the region within the orbit of the sun is practically flat, and the distortion becomes perceptible only at or beyond the distance of the sun.

H. C. VAN DE HULST, Leiden Observatory, Leiden, Netherlands: Let me also answer the preceding questions. The data in the northern sky taken in Holland [G. Westerhout, "The distribution of atomic hydrogen in the outer parts of the galactic system," Bull. Astron. Soc. Neth. No. 475 (1957)] have indicated that in the interior part of the galactic system everything is almost plane; most of the arms do not deviate by more than $25 \mathrm{pc}$ from the plane, but in the outer part they curve rather suddenly up. With respect to Ambartsumian's question, it does not make a significant difference whether the point of maximum density or the mean $Z$ coordinate is taken. 